Annales Geophysicae (2002) 20: 1105-1115 C European Geophysical Society 2002

\title{
Solar activity monitoring and forecasting capabilities at Big Bear Solar Observatory
}

\author{
P. T. Gallagher ${ }^{1,2}$, C. Denker ${ }^{2}$, V. Yurchyshyn ${ }^{2}$, T. Spirock ${ }^{2}$, J. Qiu ${ }^{2}$, H. Wang ${ }^{2}$, and P. R. Goode ${ }^{2}$ \\ ${ }^{1}$ L-3 Com Analytics Corp., NASA Goddard Space Flight Center, Greenbelt, MD 20771, USA \\ ${ }^{2}$ Big Bear Solar Observatory, New Jersey Institute of Technology, Big Bear City, CA 92314, USA
}

Received: 21 August 2001 - Revised: 15 April 2002 - Accepted: 24 April 2002

\begin{abstract}
The availability of full-disk, high-resolution $\mathrm{H} \alpha$ images from Big Bear Solar Observatory (USA), Kanzelhöhe Solar Observatory (Austria), and Yunnan Astronomical Observatory (China) allows for the continual monitoring of solar activity with unprecedented spatial and temporal resolution. Typically, this Global $\mathrm{H} \alpha$ Network (GHN) provides almost uninterrupted $\mathrm{H} \alpha$ images with a cadence of 1 min and an image scale of $1^{\prime \prime}$ per pixel.

Every hour, GHN images are transferred to the web-based BBSO Active Region Monitor (ARM; www.bbso.njit. edu/arm), which includes the most recent EUV, continuum, and magnetogram data from the Solar and Heliospheric Observatory, together with magnetograms from the Global Oscillation Network Group. ARM also includes a variety of active region properties from the National Oceanic and Atmospheric Administration's Space Environment Center, such as up-to-date active region positions, GOES 5-min X-ray data, and flare identification.
\end{abstract}

Stokes I, V, Q, and U images are available from the recently operational BBSO Digital Vector Magnetograph and the Vector Magnetograph at the Huairou Solar Observing Station of Beijing Observatory. Vector magnetograms provide complete information on the photospheric magnetic field, and allow for magnetic flux gradients, electric currents, and shear forces to be calculated: these measurements are extremely sensitive to conditions resulting in flaring activity.

Furthermore, we have developed a Flare Prediction System which estimates the probability for each region to produce C-, M-, or X-class flares based on nearly eight years of NOAA data from cycle 22. This, in addition to BBSO's daily solar activity reports, has proven a useful resource for activity forecasting.

Key words. Solar physics, astronomy and astrophysics (flares and mass ejections; instruments and techniques; photosphere and chromosphere)

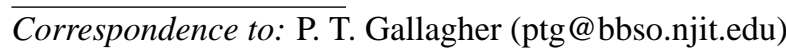

\section{Introduction}

Big Bear Solar Observatory (BBSO) has a long tradition of developing innovative instruments and interpreting data on solar activity and space weather. BBSO's lake-side location at an altitude of $2067 \mathrm{~m}$, and its excellent seeing conditions (Goode et al., 2000), together with its sophisticated instrumentation and large team of experienced scientific and technical staff, make BBSO one of the world's most ideal sites for ground-based space weather research. This, in addition to the almost real-time availability of both ground- and space-based images of the photosphere, chromosphere, and corona, make scientific activity forecasting a timely and much needed resource for the solar physics and space weather communities.

In collaboration with Kanzelhöhe Solar Observatory (KSO) in Austria and Yunnan Astronomical Observatory (YNAO) in China, BBSO has developed a high-resolution, full-disk Global $\mathrm{H} \alpha$ Network (GHN) of observatories which provide $2^{\prime \prime}$ resolution images of the solar chromosphere with up to a $30 \mathrm{~s}$ cadence. These images, when used in combination with longitudinal magnetograms and continuum images from the Michelson Doppler Imager (MDI), EUV images from the Extreme-ultraviolet Imaging Telescope (EIT) on board the Solar and Heliospheric Observatory (SOHO), and full-disk X-ray images from the Soft X-ray Telescope (SXT) on board Yohkoh further add to our ability to monitor active region evolution and make accurate predictions of solar activity. Also of key importance to this effort, is the prompt availability of high-sensitivity vector magnetograms from BBSO's Digital Vector Magnetograph (DVMG) and the vector magnetograph (VMG) of the Huairou Solar Observing Station (HSOS) of Beijing Observatory in China. Unlike longitudinal magnetograms, vector magnetograms allow for detailed properties such as shear, vertical current density, magnetic field gradients, and region fractal dimension to be measured.

During solar maximum, flares, filament eruptions, and coronal mass ejections (CME) can result in conditions which influence the performance of space- and ground-based sys- 


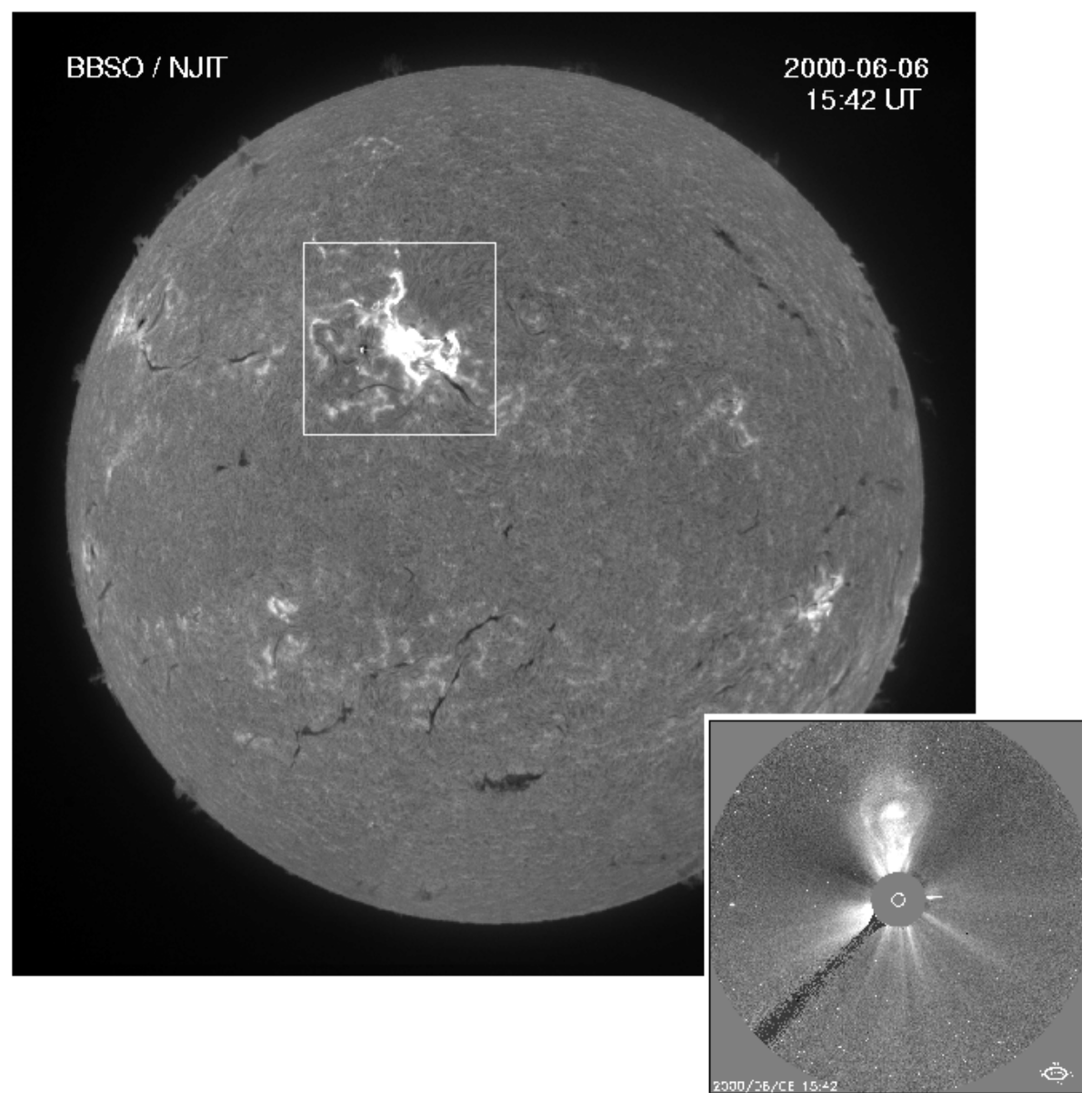

Fig. 1. Contrast enhanced full-disk $\mathrm{H} \alpha$ image obtained after dark, flatfield, and limb darkening correction. The image was recorded with BBSO's $15 \mathrm{~cm}$ Singer telescope on 6 June 2000 at 15:42 UT during the eruption of an X2.3 flare in NOAA 9026. The flare, whose position is marked by the white box, started at 14:48 UT and was associated with a halo CME and a severe magnetic storm. The inset in the lower right corner, shows the associated CME in a Large Angle and Spectrometric Coronagraph (LASCO) C3 image recorded at the same time.

tems and which can endanger humans in space and the nearEarth environment. This is particularly important today due to the increased number of commercial trans-polar aircraft flights and the increase in extra-vehicular astronaut activity required during the assembly of the International Space Station. This, therefore, prompts the need for real-time solar activity monitoring, not only to view current solar conditions, but also to make reliable, scientific forecasts of future solar activity. With this in mind, we have developed the BBSO Active Region Monitor (ARM), which provides near real-time solar data and activity forecasting.

This paper is organized as follows: in Sect. 2, the Global $\mathrm{H} \alpha$ Network and the newly developed vector magnetographs at BBSO and HSOS are described, while Sect. 3 details the web-based Active Region Monitor. Section 4 then summarizes recent results from the GHN, with our conclusions and future capabilities at BBSO then given in Sect. 5.

\section{Instrumentation}

\subsection{Global $\mathrm{H} \alpha$ network}

A new global network for high-resolution $\mathrm{H} \alpha$, full-disk observations of the Sun has been established at Big Bear Solar
Observatory (BBSO), Kanzelhöhe Solar Observatory (KSO) in Austria, and Yunnan Astronomical Observatory (YNAO) in China (Steinegger et al., 2000). Each of the three stations have $\sim 2048 \times 2048$ pixel CCD cameras to monitor the Sun with a image scale of $1^{\prime \prime}$ and a cadence of at least one image per minute. In the case of rapid solar activity change, this cadence can be increased to several images per minute. BBSO has a long tradition in obtaining synoptic full-disk observations of the Sun in $\mathrm{H} \alpha$ (Denker et al., 1999). The same applies to KSO, where high-cadence, full-disk $\mathrm{H} \alpha$ data have been obtained for more than 25 years (Otruba, 1999). All data obtained by the network are processed in exactly the same way in order to produce a homogeneous data set. After correcting for dark and flat-field images, the average quiet Sun limb darkening function is subtracted to obtain contrast-enhanced images, such as that given in Fig. 1. This typical high-resolution, full-disk $\mathrm{H} \alpha$ image was taken on 6 June 2000 at 15:42 UT, using the 15-cm Singer telescope at BBSO. The white box in Fig. 1 shows the position of an X2.3 flare which occurred in NOAA 9026, and which was associated with a halo CME, as shown by the inserted Large Angle and Spectrometric Coronagraph which is on board the SOHO spacecraft. Indeed, in combination with space- and groundbased observations, $\mathrm{H} \alpha$ full-disk observations of high temporal and spatial resolution have proven to be a key diagnostic 

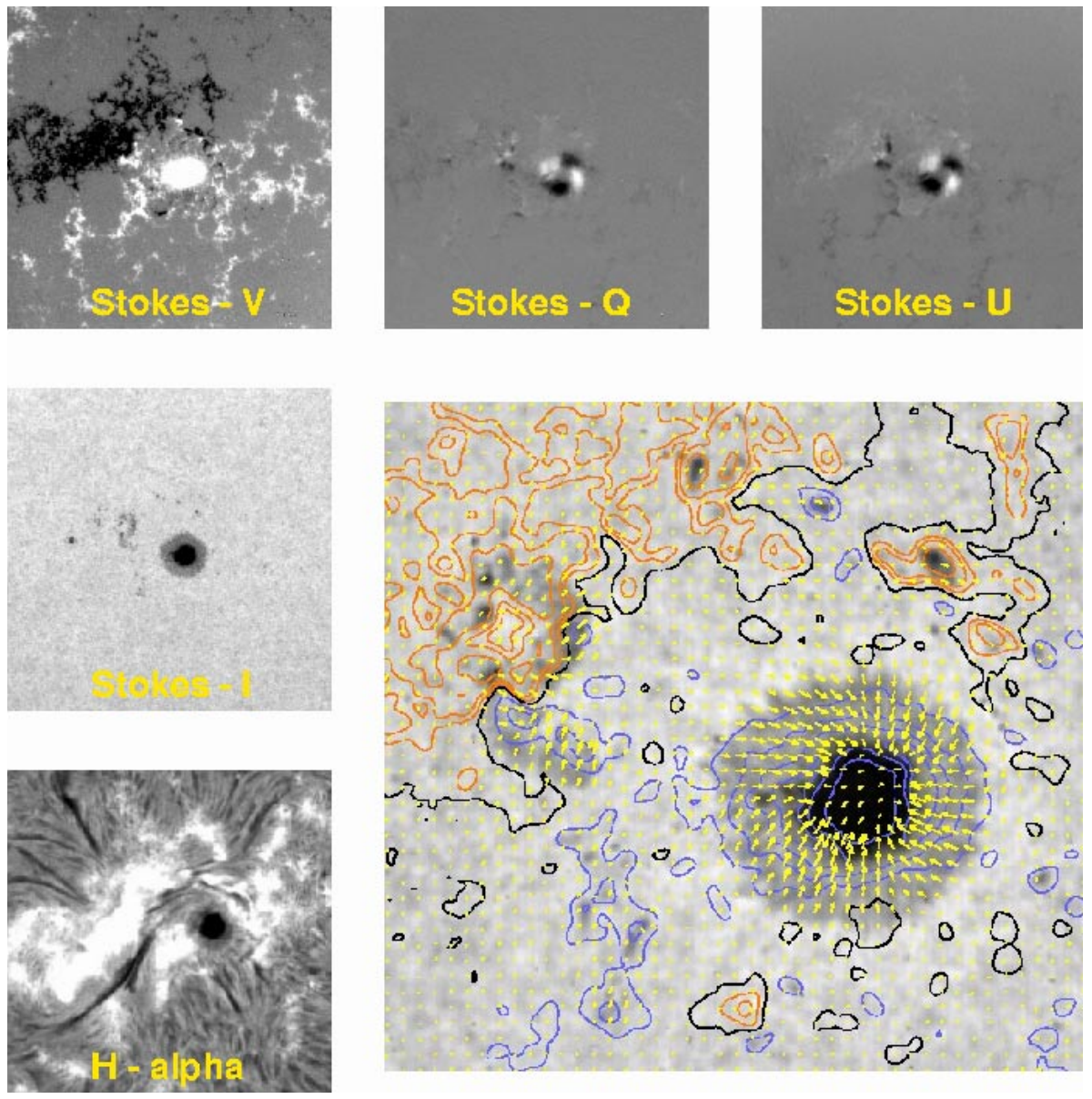

Fig. 2. Vector magnetogram of NOAA 9240 from the BBSO DVMG. The orange/blue contours indicate the positive/negative longitudinal magnetic field strength, the contour levels being $0.2,0.4,0.6$, and 0.8 of the maximum field strength. The dark solid lines indicate the longitudinal field inversion lines, while the yellow arrows indicate the strength and azimuth of the transverse magnetic field. of active regions in the photosphere, chromosphere, transition region, and corona.

The greatest time difference between the network sites is about $9.4 \mathrm{~h}$ between BBSO and YNAO. The difference between BBSO and KSO is about $8.7 \mathrm{~h}$, and between YNAO and KSO only $5.9 \mathrm{~h}$. During the Northern Hemisphere summer, each station can observe for $12 \mathrm{~h}$ on clear days, therefore, leaving no nighttime gaps. During the Northern Hemisphere winter, when each station is expected to operate for $8 \mathrm{~h}$ per day, the BBSO/YNAO gap is about $1.4 \mathrm{~h}$, while the BBSO/KSO gap about $0.7 \mathrm{~h}$. Based on the weather records for the three stations, a duty cycle of $\sim 70 \%$ in summer and $\sim 60 \%$ in winter is expected.

\subsection{Digital Vector Magnetograph Network}

The Digital Vector Magnetograph (DVMG) at BBSO is a filter-based magnetograph mounted on the $25 \mathrm{~cm}$ vacuum refractor telescope at BBSO (Varsik, 1995; Spirock et al., 2001). The pre-filter has a central wavelength of $610 \mathrm{~nm}$, a bandwidth of $1.4 \mathrm{~nm}$ and a transmission of about $75 \%$. The polarization optics consist of two nematic liquid crystal variable retarders, used to select a particular Stokes vector, and a single $1 / 2$ waveplate ferroelectric liquid crystal, used as the fast switcher. The main filter is a Zeiss $\mathrm{H} \alpha$ filter tuned to the magnetically sensitive Ca I absorption line at $610.3 \mathrm{~nm}$. The resulting light is then focused onto a Silicon Mountain Design 1M15, 12-bit, $1024 \times 1024$ pixel, CCD camera with an $18 \%$ maximum quantum efficiency. The camera is normally run in $512 \times 512$ pixel mode with a cadence of 30 frames per second and a field-of-view of $315^{\prime \prime} \times 315^{\prime \prime}$.

The vector magnetograph (VMG) at HSOS forms the second site of our vector magnetograph network, thus allowing us to monitor active region fields with only 4 to $8 \mathrm{~h}$ night gaps, depending on the season. The VMG was developed by Ai (1987) and is very similar to the older BBSO digital magnetograph system described in Wang et al. (1998), except that it uses the Fe I $532.4 \mathrm{~nm}$ line $\left(g_{L}=1.5\right)$. Like the BBSO DVMG, the temporal and image scale of the VMG depends on the number of frames added to form a single magnetogram.

The BBSO DVMG and HSOS VMG data sets consists of narrow-band images in each of the four Stokes parameters: $I$, the intensity; $V$, the difference between left- and right-hand circular polarized images; $U$, the difference between two orthogonal linear polarized images; and $Q$, the same as $U$, but with a $45^{\circ}$ change in the azimuth angle. The amplitude of the transverse field is then determined by

$$
B_{T}=C \times\left(Q^{2}+U^{2}\right)^{\frac{1}{4}},
$$


where $C$ is a calibration constant, and the field's azimuthal angle is determined by:

$\theta=\frac{1}{2} \arctan \left(\frac{U}{Q}\right)$.

Figure 2 gives a BBSO DVMG magnetogram of NOAA 9240, together with an $\mathrm{H} \alpha$ images of the region for comparison.

Vector magnetograms can then be used to establish several parameters to aid in flare prediction. These include:

- Integrated Magnetic Shear:

There are several definitions of the term "magnetic shear", the first due to the NASA Marshall Space Flight Center group. We define the shear as the product of the shear angle and the measured transverse magnetic field (Wang, 1992). Specifically, the mean shear angle is defined as

$$
\bar{\theta}=\frac{\sum\left|B_{T}\right| \theta}{\sum\left|B_{T}\right|}
$$

where $B_{T}$ is the measured transverse field strength and $\theta$ is the angle difference between the azimuthal angle of the measured transverse field and the transverse of the potential component calculated using the measured longitudinal field as the boundary condition.

\section{- Vertical Current Density:}

Using the horizontal distribution of the transverse components of the magnetic field allows for the vertical current density to be calculated via

$J_{z}=\frac{1}{\mu_{0}}\left(\frac{\partial B_{y}}{\partial x}-\frac{\partial B_{x}}{\partial y}\right)$

where $J_{z}$ is in units of $\mathrm{A} \mathrm{m}^{-2}$ and $\mu_{0}=1.2 \times 10^{-2}$ $\mathrm{Gm} \mathrm{A}^{-1}$. Recently, Li et al. (2001) found an important link between the occurrence of major flares and the appearance of new currents brought up by new magnetic flux, while Falconer (2001) found a close connection between the occurrence of major flares, currents, and the length of the sheared neutral line in active regions.

- Magnetic Field Gradients:

Gradients in the longitudinal magnetic field in the photosphere have long been known to be related to flaring activity in active regions (Zhang et al., 1994). We focus our analysis on the magnitude of the gradient of the line-of-sight field which can quite simply be calculated using,

$$
|\nabla B|=\sqrt{\left(\frac{\partial B}{\partial x}\right)^{2}+\left(\frac{\partial B}{\partial y}\right)^{2}} .
$$

\section{- Fractal Dimension:}

The distribution of the photospheric magnetic field is extremely complex, its study providing important information on the state and evolution of active region fields (Meunier, 1999). Therefore, we use the fractal dimension to characterize the spatial complexity of the longitudinal field configuration using the standard perimeter/area method described by Mandelbrot (1983). Specifically, the fractal dimension $d$, is given by

$$
d=2 \frac{\Delta \log P}{\Delta \log A},
$$

which corresponds to twice the slope of the variation of perimeter $P$ with area $A$ on a $\log / \log$ scale. If the active regions were a perfect circle, or square, then $d$ would be unity, while $d$ tends to 2 as the region increases in complexity.

We are also in the process of adding the Crimean Observatory in the Ukraine to the BBSO and HSOS vector magnetograph collaboration. This will for the first time create a global vector magnetograph network which will complement full-disk data from GHN with a similar duty cycle.

\subsection{Near real-time image reconstruction}

At BBSO, we have also developed a system for near realtime image reconstruction which features a combination of frame selection, speckle masking imaging, and parallel computing (Denker et al., 2001). The current system is based on a 4 node $(500 \mathrm{MHz})$ Beowulf cluster and provides diffraction limited, $256 \times 256$ pixel images at 1 min cadence. Since the software is scalable, it is simple to upgrade to a larger Beowulf cluster, e.g. a 32 node machine at $1 \mathrm{GHz}$ is capable of reconstructing a $1024 \times 1024$ pixel image in $1 \mathrm{~min}$. The corresponding field-of-view is about $80^{\prime \prime} \times 80^{\prime \prime}$, which allows us to study the dynamics of active regions. One application in the context of space weather forecasts is to combine image reconstruction with Local Correlation Tracking (LCT) and derive horizontal flow fields.

Many major flares are associated with new flux emergence inside sunspots (Zirin and Wang, 1993). The new flux appears in an elongated mixed channel structure visible in white-light images. Complicated magnetic configurations are likely to produce both large- and small-scale activity. There are two very important aspects of the evolution of active regions which may be closely associated with solar flares: (1) new flux emergence and (2) unusual flow motions. Denker and Wang (1998) demonstrated the power of diffraction limited observations in a flaring active region. NOAA 8076 was one of the first active regions of cycle 23 and was observed by us for the first time with high-spatial resolution white-light observations on 31 August 1997. The almost diffraction limited speckle reconstruction given in Fig. 3 reveals the complex and highly dynamic behavior of a small emerging configuration in the central part of NOAA 8076. 


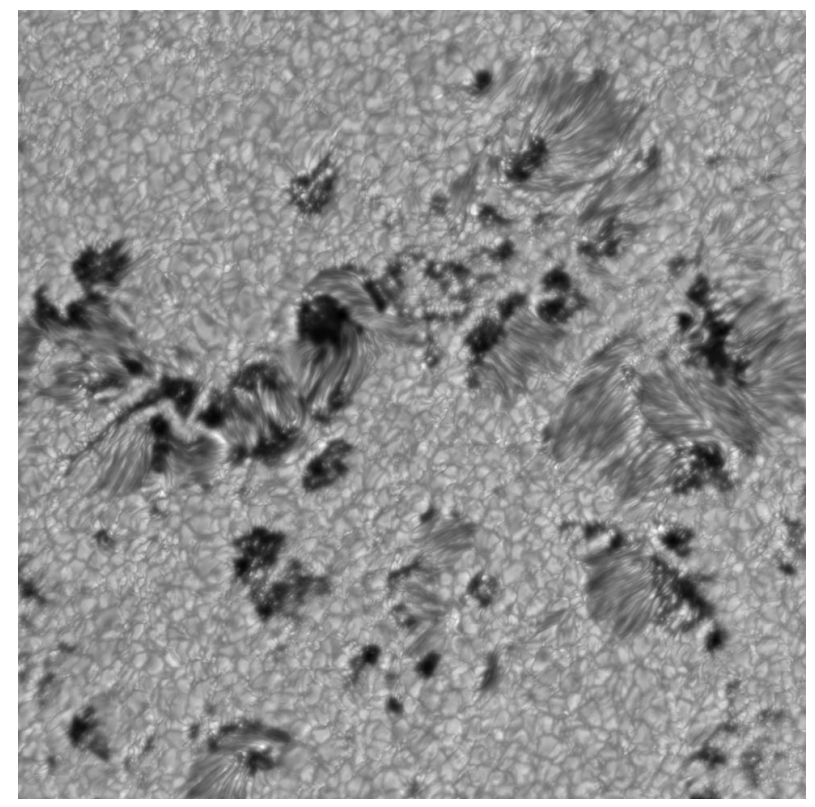

Fig. 3. White-light speckle reconstruction $(520 \mathrm{~nm})$ of NOAA 8674 obtained on 27 August 1999 with the $65 \mathrm{~cm}$ vacuum reflector at BBSO.

We found strong shear flows and indications of strong transverse fields in the small spot.

\section{Active Region Monitor}

The Active Region Monitor (ARM) is a web-based solar activity monitoring tool which provides the most recent images from a variety of ground- and space-based solar observatories and can be found at www.bbso.njit.edu/arm. ARM was developed within Research System's Interactive Data Language (IDL), using the current version of the SolarSoftWare (SSW; www . lmsal . com/s sw) tree (Freeland and Handy, 1998).

In addition to providing images from the photosphere, chromosphere, and corona, the ARM pages include tables of active region data from NOAA, including up-to-date active region positions. Furthermore, a Flare Prediction System (FPS) has been included, which provides a table of region names, together with flaring probabilities. Further detail on these, and other features of ARM are discussed below.

\subsection{Full-disk images}

ARM displays full-disk images from GONG+, GHN, magnetograms, and continuum and EUV images from MDI and EIT. On the hour, ARM connects to the BBSO data archive at $\mathrm{ftp} / / / \mathrm{ftp}$.bbso.njit.edu and locates the most recent full-disk $\mathrm{H} \alpha$ image. When located, this file is then transferred via FTP to the local machine on which ARM is running.

EIT (Delaboudinière et al., 1995) provides full-disk EUV images in narrow bands centered on He II (30.4 nm), Fe IX/X

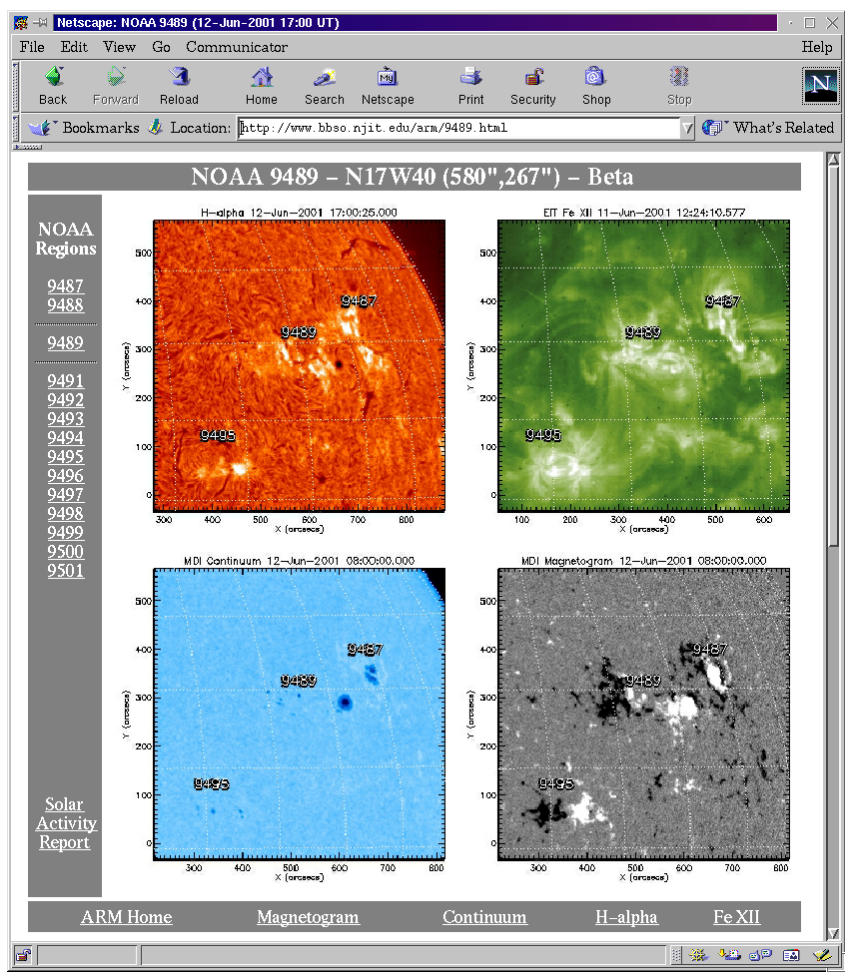

Fig. 4. Snap-shot from ARM showing a $10^{\prime} \times 10^{\prime}$ zoom of NOAA active region 9489. Clockwise from top left, ARM shows BBSO $\mathrm{H} \alpha$, EIT Fe XII, MDI magnetogram, and MDI continuum.

$(17.1 \mathrm{~nm})$, Fe XII $(19.5 \mathrm{~nm})$ and Fe XV $(28.4 \mathrm{~nm})$ with a $45^{\prime} \times 45^{\prime}$ field-of-view and $2.6^{\prime \prime}$ pixels. On the hour, the ARM software transfers the most recent Fe XII $(19.5 \mathrm{~nm})$ and Fe XV (28.4 nm) FITS file from the SOHO archive, and then automatically background subtracts, degrids, and flat-fields the data.

MDI (Scherrer et al., 1995) records high resolution magnetograms, dopplergrams, and line-depth images of the photosphere in the Ni I $676.8 \mathrm{~nm}$ absorption line with a spatial resolution of $2^{\prime \prime}$ per pixel. On the hour, the ARM software also transfers the most recent continuum image and magnetogram from the SOHO archive. The continuum image is then flat-fielded and corrected for limb darkening, while the magnetogram is scaled appropriately.

Full-disk longitudinal magnetograms are also sourced from four of the six sites of the Global Oscillation Network Group (Harvey et al., 1996). Namely, data is obtained from: Big Bear, California; Mauna Loa, Hawaii; Learmonth, Western Australia; Cerro Tololo, Chile. The longitudinal magnetograms are in turn used to derive magnetic field gradient maps using Eq. (5).

The calibrated data from GONG+, GHN and EIT are then overlayed with Stonyhurst grids using SSW mapping software, while active regions are identified and labelled using positions from NOAA.

A $10^{\prime} \times 10^{\prime}$ field centered on each active region is also extracted from each full-disk image and written to the ARM 
web pages. A zoom view of NOAA 9489 from 12 June 2001 is given in Fig. 4.

\subsection{Active region tables}

Active region properties, such as area in millionths of the solar hemisphere, McIntosh and Mount Wilson classifications, and total number of constituent spots, are obtained from the Solar Region Summary issued by the NOAA Space Environment Center at 00:30 UT on a daily basis. The region positions, obtained from this report, are then rotated differentially to the current time and are tabulated as both heliographic and heliocentric positions. ARM is thus the only source of active region positions which are valid to the nearest hour.

\subsection{Flare prediction system}

Similarly to forecasters at NOAA's Space Environment Center, we have developed a flare prediction system based on the McIntosh active region classification scheme (McIntosh, 1990). The McIntosh system describes the fundamental properties using three parameters: $Z$ gives the Modified Zürich Class; $p$ describes the penumbra of the largest spot; $c$ gives the sunspot distribution. There have been several attempts in the past to calculate flare rates from active region flaring probabilities using both the Mount Wilson (Sammis et al., 2000) and the McIntosh classification schemes (McIntosh, 1990; Bornmann et al., 1994; Bornmann and Shaw, 1994). Our system uses almost eight years of SEC flare occurrence and active region McIntosh classification data from November 1988 to June 1996, thus sampling the majority of activity in cycle 22. Following Kildahl (1980), and average flare rate is calculated based on tables of daily average flare rates. For example, between November 1988 and June 1996, there were 302 regions with a McIntosh classification Eai. Since this class produced 172 C-class events, $62 \mathrm{M}$-class events, and $2 \mathrm{X}$-class events, a flare rate can be estimated by dividing the number of events by the number of occurrence of this class, i.e. the average daily flare rate of M-class flares for an Eai region is $\sim 62 / 302$ or $\sim 0.21$ flares per day.

The frequency of occurrence of solar flares follows Poisson statistics (Bornmann et al., 1994), and so knowing the average flare rate allows us to calculate the probability for a region of a particular class to produce one or more C-, $\mathrm{M}$ - or $\mathrm{X}$-class event for a given 24-h period.

The probability of observing $N$ events per unit time interval is given by the basic Poisson distribution:

$P_{\mu}(N)=\frac{\mu^{N}}{N !} \exp (-\mu)$

where $\mu$ is the average number of events expected in each time interval. The probability of observing one or more events in a one day (24-h) period can then be estimated via

$P_{\mu}(N \geq 1)=1-P_{\mu}(N=0)$,
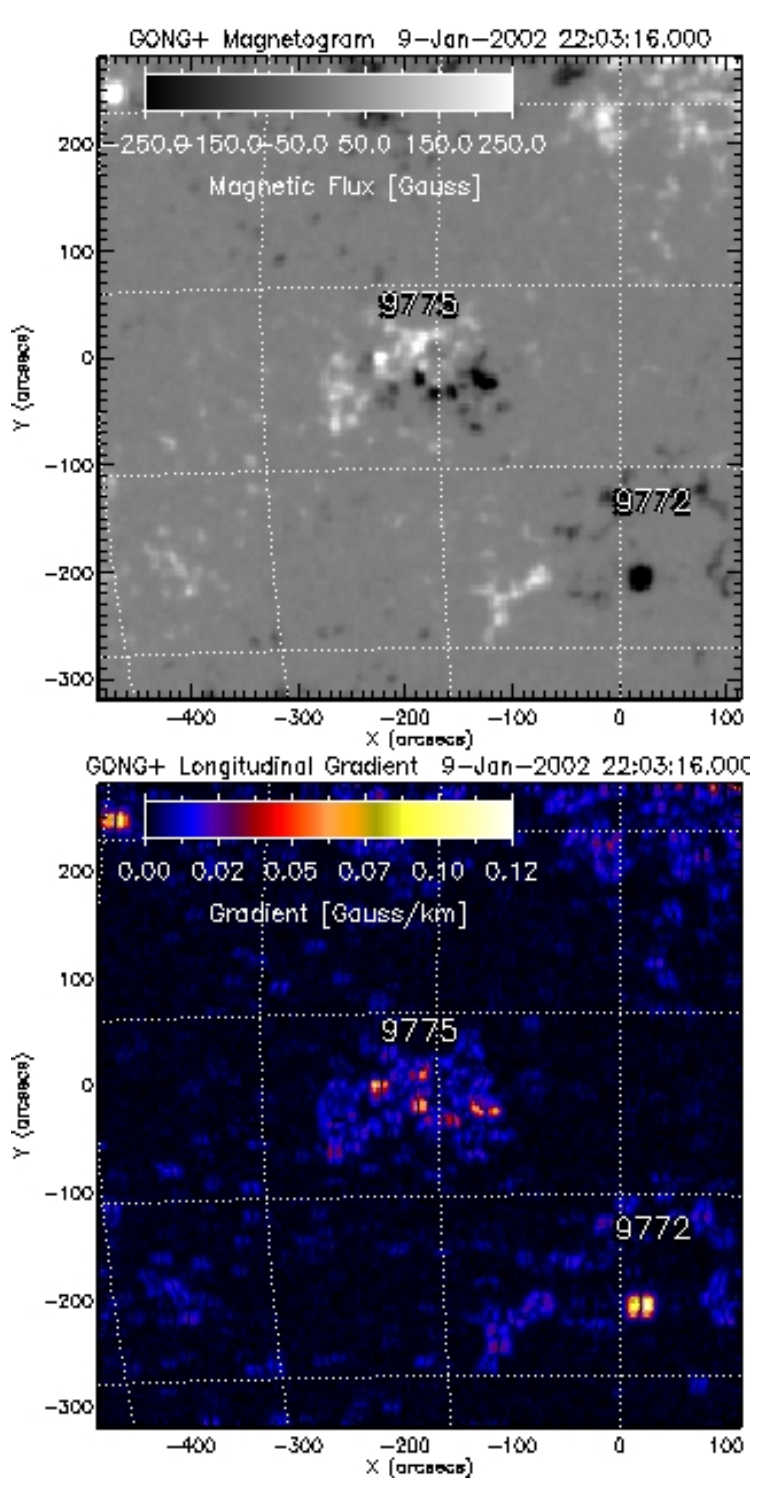

Fig. 5. The top panel gives a GONG+ magnetogram taken at 22:03:16 UT on 9 January 2002, while the derived gradient of the longitudinal magnetic field is given at bottom.

where $P(N=0)$ is the probability of no events occurring. More explicitly, the probability for observing one or more events in a $24-\mathrm{h}$ period is given by:

$P_{\mu}(N \geq 1)=1-\exp (-\mu)$.

\subsection{Solar activity reports and warnings}

Once per day, BBSO issues a solar activity report providing information on all the active regions on the Sun, including the probability for flare events. In addition, solar activity warnings are posted on the ARM web pages and distributed immediately to an e-mail list. These warnings have replaced BEARALERTS (Zirin and Marquette, 1991) as of 24 November 1998. Requests for subscription 
to the activity warning e-mail distribution list can be sent to: activity@bbso.njit.edu.

\section{Recent results}

The GHN has been in operation for just over a year and has produced a number of important results. The BBSO and HSOS vector magnetographs, on the other hand, have only been operational for half this time, and in addition to ARM, have already proven crucial to solar activity forecasting. In this section, we detail some of the recent results from these systems.

\subsection{Flare associated magnetic gradients}

There have been several attempts to derive properties of the photospheric magnetic field associated with flaring activity such as shear, electric current, and field gradient. The top panel of Fig. 5 shows a $10^{\prime \prime} \times 10^{\prime \prime}$ image extracted from a full-disk GONG+ magnetogram obtained on 9 January 2002. The data have been scaled to \pm 250 Gauss to highlight weak features which would otherwise have been difficult, if not impossible, to observe in the magnetogram. Also labelled in the image are two simple active regions: NOAA 9772 is an $\alpha$ region and NOAA 9775, a $\beta$. The bottom panel of Fig. 5 gives the gradient of the magnetic field derived using Eq. (5). As expected, the solitary spot of NOAA 9772 shows the largest gradients $(\sim 12$ Gauss $/ \mathrm{km})$ due to the significant magnetic flux difference between the spot center and its boundaries. NOAA 9775, on the other hand, consists of numerous smaller spots, each being identifiable in the gradient map as small, isolated double-peaks with gradients of $\leq 0.07$ Gauss $/ \mathrm{km}$.

In contrast to these simple regions, the top left panel of Fig. 6, shows a $10^{\prime \prime} \times 10^{\prime \prime}$ magnetogram centered on the large complex region NOAA 9773. This data was again extracted from a full-disk GONG+ magnetogram taken on 9 January 2002 and is scaled to \pm 250 Gauss. This region first became visible on the Sun on 4 January and developed rapidly from a simple $\alpha$ spot to a complex region consisting of some twenty constituent spots on 9 January, when the data analyzed here were obtained. On this date, the region was classified as a $\beta \gamma \delta$ region with a distinct inversion line within the region's interior. The top right panel shows the gradient of the magnetic field (in Gauss $/ \mathrm{km}$ ) derived from this magnetogram. It is clear there are strong magnetic gradients of up to $0.22 \mathrm{Gauss} / \mathrm{km}$ visible across the inversion line of the delta, which only became visible on this day: magnetograms from the preceding and subsequent days displayed gradients of less than $\sim 0.12 \mathrm{Gauss} / \mathrm{km}$. Indeed, the majority of active regions typically show gradients of less than $\sim 12$ Gauss $/ \mathrm{km}$, with only the most flare productive regions showing larger, more distinct gradients. The bottom panel of Fig. 6 shows the GOES X-ray activity for 8 and 9 January 2002, showing the increase in flaring activity from the end of 8 January, when the magnetic gradient measurements presented here were obtained.

The fact that large gradients are observed in regions showing opposite polarity mixing is, of course, not too surprising if we consider the terms on the right-hand side of Eq. (5), i.e. fields in close proximity (i.e. with small $\partial x$ and/or $\partial y$ ) which have large, oppositely polarized magnetic fluxes (i.e. large $\partial B$ ) will result in large field gradients. Further examples of the relationship between magnetic flux gradients and flare productivity and their physical interpretation will be discussed in a follow-on paper which is currently in preparation (Gallagher et al., 2002).

\subsection{The largest particle event in cycle 23}

Coronal mass ejections (CMEs) are often associated with erupting magnetic structures or disappearing filaments. The majority of CMEs headed directly toward the Earth are observed at $1 \mathrm{AU}$ as magnetic clouds, the region in the solar wind where the magnetic field strength is higher than average and there is a smooth rotation of the magnetic field vectors. The three-dimensional structure of magnetic clouds can be represented by a force-free flux rope. When CMEs reach the Earth, they may or may not cause magnetic storms, alter the Earth's magnetic field, or produce the phenomena known as auroras. The geoeffectiveness of a solar CME depends on the orientation of the magnetic field in it (Yurchyshyn et al., 2001).

One of the largest and the most powerful events of cycle 23 occurred in active region NOAA 9077 on 14 July 2000. The eruption started at approximately 09:30 UT with the activation of a low lying dark filament visible in TRACE and in $\mathrm{H} \alpha$ images, as indicated by the arrow in Fig. 7 (see also Zhang et al., 2001). Presumably, the filament eruption led to the destabilization of the large X-ray loop: the top right image in Fig. 7 shows the post-flare magnetic configuration, where the X-ray loop is no longer seen. This loop was then replaced by a post-flare loop system visible in the 01:01 UT Yohkoh and 10:33 UT TRACE images.

Two bottom panels of Fig. 7 show the HSOS magnetogram taken several hours before the flare, and a linear force-free field extrapolation with $\alpha=-0.019 \mathrm{arc} / \mathrm{s}$. Due to the limitations of linear force-free models, we failed to repeat the observed large-scale coronal field in detail. A comparison of the calculated field lines with the 09:27 UT Yohkoh image shows that the observed field is far more twisted than the modelled field. Normally, $|\alpha| \leq 0.02 \mathrm{arc} / \mathrm{s}$ is enough to fit the large-scale field of an active region. On the other hand, the model with negative helicity matches the post-flare loops system seen in the 10:33 UT TRACE image ${ }^{1}$. When we look at the observed post-flare loop system from the positive polarity side, we find that the magnetic field in both the TRACE and modelled loops is rightward directed. According to Mar-

\footnotetext{
${ }^{1}$ Based upon this, we estimate the density of electric currents in the post-flare loops: the extrapolated magnetic field strength is about $300 \mathrm{G}$, which implies a current density of $0.006 \mathrm{~A} \mathrm{~m}^{-2}$.
} 


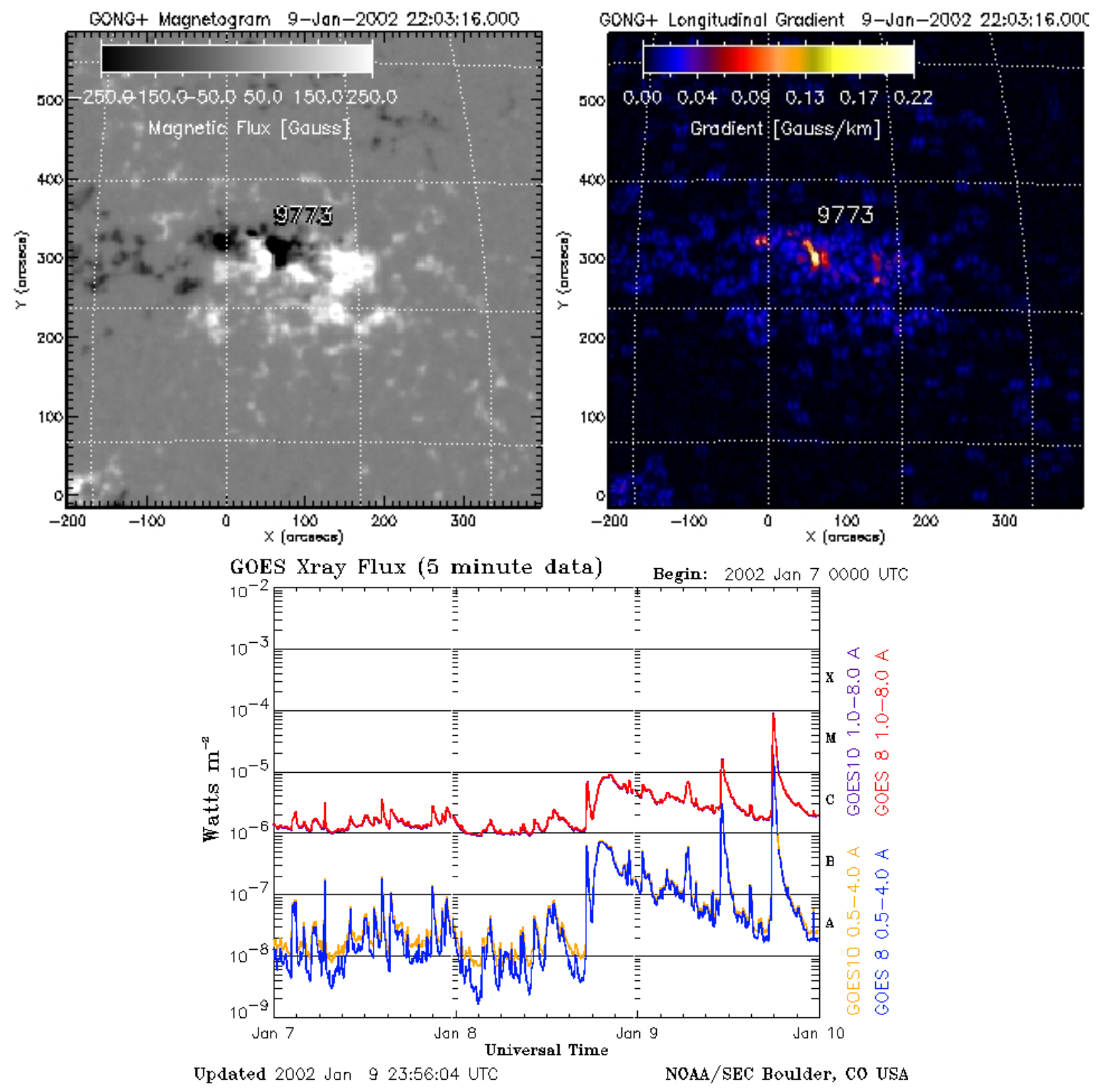

Fig. 6. The top left panel gives a GONG+ magnetogram taken at 22:03:16 UT on 9 January 2002, while the top right panel gives the gradient of the longitudinal magnetic field. A three-day GOES X-ray plot for 7-9 January 2002 is given at bottom.

tin et al. (1994), this implies that the post-flare system is dextral or it has negative helicity, which can be confirmed from the large reverse-S shaped X-ray loop in the upper-left panel of Fig. 7. In the HSOS magnetogram, the location of the large X-ray loop seen before the eruption is indicated with a bold white curve. Comparison of the Yohkoh/SXT image and HSOS magnetogram shows, that the axial field in the large $\mathrm{X}$-ray loop was west-east directed, which coincides with the model calculations.

This two-ribbon flare was followed by a fast halo CME which had a mean velocity of about $1500 \mathrm{~km} / \mathrm{s}$, and according to the SOHO/CELIAS/MTOF Proton Monitor ${ }^{2}$, its arrival was preceded by a strong interplanetary shock (see also Klein and Burlaga, 1982; Vandas et al., 1997). The CME ar-

\footnotetext{
2 http://sohowww.nascom.nasa.gov/data/CME/20000714/
}

rival was also accompanied by a severe magnetic storm during which the $D_{s t}$ index decreased to -300 (see Fig. 8, bottom panel). The figure also plots 1-h averages of the IMF at about $0.99 \mathrm{AU}$ from the Sun. A magnetic cloud (MC) is identified by the rapid growth of the magnetic field on 15 July at about 14:00 UT. Upon arrival of the MC at $t_{1}$, the $B_{z}$ component was directed southward, and it rotates during the passage of the satellite through the MC (Fig. ACE, $X-Z$ plot). At the same time, the signs of the $B_{x}$ and $B_{y}$ components do not change ( $Y-Z$ and $X-Y$ plots). Since the $B_{z}$ component rotates, it implies that $B_{Z}$ is the azimuthal component of the flux rope's magnetic field, and the axis of the flux rope is oriented in the east-west direction. The $B_{z}$ component vanishes on 16 July, at $t_{2} \approx 00: 00 \mathrm{UT}$, which suggests that the axis of the flux tube is nearly in the plane of the ecliptic, i.e. it runs 


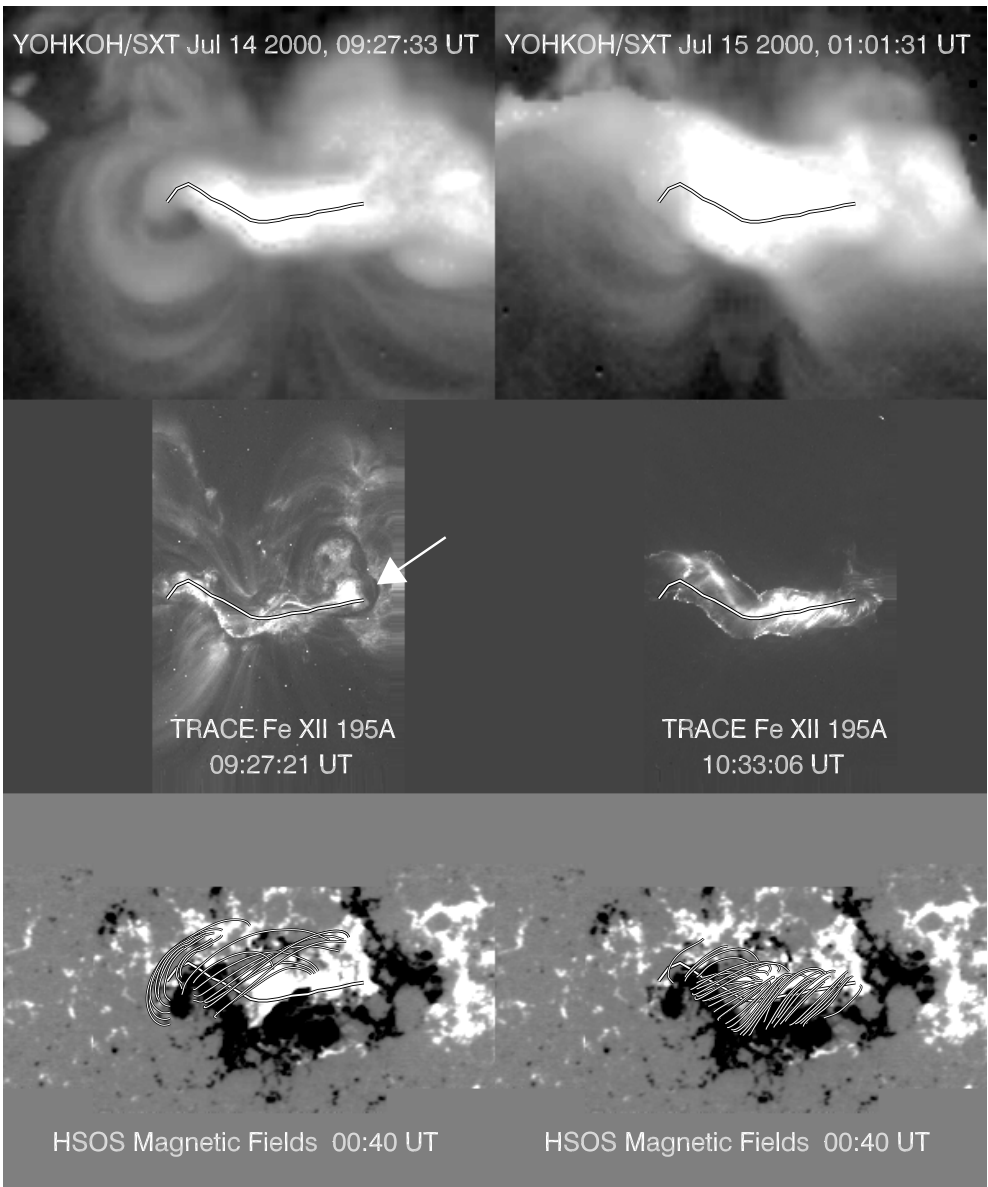

Time, UT

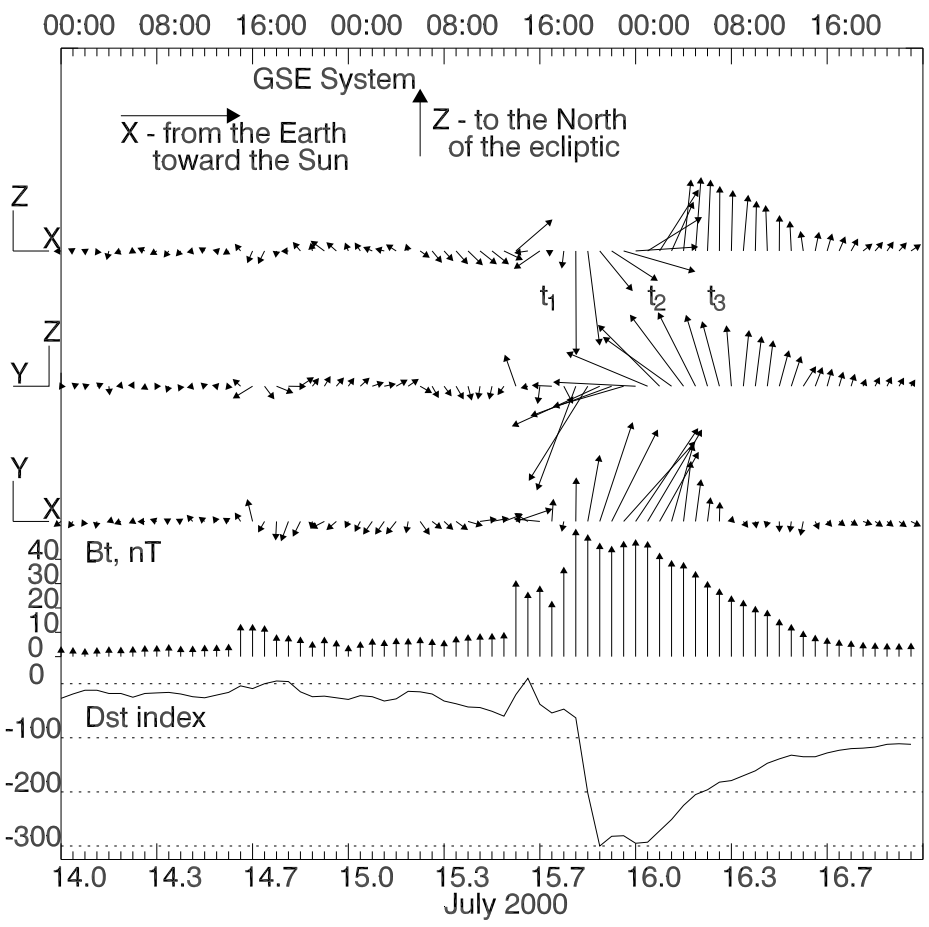

Fig. 7. Yohkoh and TRACE pre- and post-flare images of the 14 July 2000 two-ribbon flare. Bottom: HSOS longitudinal field overlaid with a linear force-free field lines. North - to the top, west - to the right.
Fig. 8. Plot of 1-h averages of the IMF in the 15 July 2000 MC near the Lagrange point, L1. Magnetic field components are plotted in the GSE system, where $X$-axis points from the Earth toward the Sun, $Y$ is in the ecliptic plane and negative in the direction of planetary motion, and $Z$ is parallel to the ecliptic pole. Two bottom panels show the variations of the IMF strength and the $D_{s t}$ index. 
nearly along the Sun's equator (note, that on 14 July 2000, $P_{0}=3^{\circ}$ ). The direction and helicity of the axial magnetic field can, therefore, be seen to coincide with the direction and helicity of the large X-ray loop and the entire active region as well.

\section{Conclusions}

Monitoring solar activity with a global network of full-disk $\mathrm{H} \alpha$ telescopes, vector magnetograms from BBSO and HSOS in China, in conjunction with longitudinal magnetograms, EUV and continuum images from SOHO, put us in an advantageous position for detecting and predicting changes in solar activity. For the first time, this suite of ground- and space-based instruments allow us to characterize active regions from the photosphere, through the chromosphere, to the corona. Future automatic detection of flares, filament eruptions, and other events (Gao et al., 2002) will also allow us to respond rapidly with additional observations, such as high-cadence vector magnetograms, narrow-band filtergrams, and almost diffraction limited speckle images.

Acknowledgements. This work was supported by NASA grants NAG5-9682, NAG5-9738, ONR N00014-97-11037 and by NSF grants ATM-9903515, ATM-0076602, ATM-0086999. The SOHO/LASCO data used here are produced by a consortium of the Naval Research Laboratory (USA), Max-Planck-Institut fuer Aeronomie (Germany), Laboratoire d'Astronomie (France), and the University of Birmingham (UK), while EUV images are courtacy of the EIT Consortium. SOHO is a project of international cooperation between ESA and NASA. The Yohkoh SXT instrument was developed jointly by the Lockheed-Martin Palo Alto Research Laboratory and the National Astronomical Observatory of Japan. This work utilizes data obtained by the GONG project, managed by the National Solar Observatory, which is operated by AURA, Inc. under a cooperative agreement with the National Science Foundation.

The Editor in Chief thanks two referees for their help in evaluating this paper.

\section{References}

Ai, G.: Pub. Beijing Astron. Obs., 9, 27, 1987

Bornmann, P. L., and Shaw, D.: Flare rates and the McIntosh activeregion classifications, Sol. Phys., 150, 127-146, 1994.

Bornmann, P. L., Kalmbach, D., and Kulhanek, D.: McIntosh active-region class similarities and suggestions for mergers, Sol. Phys., 150, 147-164, 1994.

Delaboudinière, J.-P., Artzner, G. E., Brunaud, J., Gabriel, A. H., Hochedez, J. F., Millier, F., Song, X. Y., Au, B., Dere, K. P., Howard, R. A., Kreplin, R., Michels, D. J., Moses, J. D., Defise, J. M., Jamar, C., Rochus, P., Chauvineau, J. P., Marioge, J. P., Catura, R. C., Lemen, J. R., Shing, L., Stern, R. A., Gurman, J. B., Neupert, W. M., Maucherat, A., Clette, F., Cugnon, P., and van Dessel, E. L.: EIT: Extreme-Ultraviolet Imaging Telescope for the SOHO Mission, Sol. Phys., 162, 291-312, 1995.

Denker, C. and Wang, H.: High Spatial Resolution Observations of a Small Delta Spot, Ap. J., 502, 493-497, 1998.

Denker, C., Johannesson, A., Marquette, W., Goode, P. R., Wang, H., and Zirin, H.: Synoptic Halpha Full-Disk Observations of the Sun from Big Bear Solar Observatory - I. Instrumentation, Image Processing, Data Products, and First Results, Sol. Phys., 184, 87-102, 1999.

Denker, C., Yang, G., and Wang. H.: Near Real-Time Image Reconstruction, Sol. Phys., 202, 63-70, 2001.

Falconer, D.: A prospective method for predicting coronal mass ejections from vector magnetograms, J. Geophys. Res., 106, $25185,2001$.

Freeland, S. L. and Handy, B. N.: Data Analysis with the SolarSoft System, Sol. Phys., 182, 497-500, 1998.

Gallagher, P. T., Moon, Y.-J., and Wang, H.: Active Region Monitoring and Flare Forecasting I. Data Processing and First Results, Sol. Phys., accepted, 2002.

Gao, J., Wang, H., and Zhou, M.: Development of an Automatic Filament Disappearance Detection System, Sol. Phys., 205, 93103, 2002.

Goode, P. R., Wang, H., Marquette, W. H., and Denker, C.: Measuring Seeing from Solar Scintillometry and the Spectral Ratio Technique, Sol. Phys., 195, 421-431, 2000.

Harvey, J. W., Hill, F., Hubbard, R., Kennedy, J. R., Leibacher, J. W., Pintar, J. A., Gilman, P. A., Noyes, R. W., Title, A. M., Toomre, J., Ulrich, R. K., Bhatnagar, A., Kennewell, J. A., Marquette, W., Patrón, J., Saa, O., and Yasukawa, E.: The Global Oscillation Network Group (GONG) Project, Science, 272, 12841286, 1996.

Kildahl, K. J. N.: in Solar-Terrestrial Predictions Proceedings, (Ed) Donnelly R. F., 3, 166, 1980.

Klein, L. W. and Burlaga, L. F.: Interplanetary magnetic clouds at 1 AU, J. Geophys. Res., 87, 613-624, 1982.

Krall, K. R., Smith, J. B., Hagyard, M. J., West, E. A., and Cummings, N. P.: Vector magnetic field evolution, energy storage, and associated photospheric velocity shear within a flareproductive active region, Sol. Phys., 79, 59-75, 1982.

Kuhn, J. R., Lin, H., and Loranz, D.: Gain calibrating nonuniform image-array data using only the image data, Pub. Astron. Soc. Pac., 103, 1097-1108, 1991.

Li, W., Lee, C. Y., Chae, J., Wang, J., Goode, P. R., Wang, H., and Ai, G.: Ap. J., submitted, 2001.

Mandelbrot, B. B.: The Fractal Geometry of Nature, New York, Freeman, 1983.

Martin, S. F., Billimoria, R., and Tracadas, P. W.: in Solar Surface Magnetism, ASI Series C, (Eds) Rutten, R. J. and Schrijver, C. J., Dordrecht, Kluwer, 433, pp. 303, 1994.

McIntosh, P. S.: The Classification of Sunspot Groups, Sol. Phys., 125, 251-267, 1990.

Meunier, N.: Fractal Analysis of Michelson Doppler Imager Magnetograms: A Contribution to the Study of the Formation of Solar Active Regions, Ap. J., 515, 801-811, 1999.

Otruba, W.: ASP Conference Series 184, 314, 1999.

Sammis, I., Tang, F., and Zirin, H.: The Dependence of Large Flare Occurrence on the Magnetic Structure of Sunspots, Ap. J., L540, 583-587, 2000.

Scherrer, P. H., Bogart, R. S., Bush, R. I., Hoeksema, J. T., Kosovichev, A. G., Schou, J., Rosenberg, W., Springer, L., Tarbell, T. D., Title, A., Wolfson, C. J., and Zayer, I.: MDI Engineering Team: Sol. Phys., 162, 129-188, 1995.

Spirock, T. J., Denker, C., Chen, J., Chae, J., Qiu, J., Varsik, J., Wang, H., Goode, P., and Marquette, W.: ASP Conference Series, in press, 2001.

Steinegger, M., et al.: ESA SP-463, 617, 2000

Tsuneta, S., Acton, L., Bruner, M., Lemen, J., Brown, W., Caravalho, R., Catura, R., Freeland, S., Jurcevich, B., and Owens, J.: 
The soft X-ray telescope for the SOLAR-A mission, Sol. Phys., 136, 37-67, 1991.

Varsik, J. R.: Calibration of the Big Bear Videomagnetograph, Sol. Phys., 161, 207-228, 1995.

Wang, H.: Evolution of vector magnetic fields and the August 27 1990 X-3 flare, Sol. Phys., 140, 85-98, 1992.

Wang, H., Denker, C., Spirock, T., Goode, P. R., Yang, S., Marquette, W. H., Varsik, J., Fear, R. J., Nenow, K., and Dingley, D. D.: New Digital Magnetograph At Big Bear Solar Observatory, Sol. Phys., 183, 1-13, 1998.

Yurchyshyn, V. B., Wang, H., Goode, P. R., and Deng, Y.: Orientation of the Magnetic Fields in Interplanetary Flux Ropes and
Solar Filaments, Ap. J., 563, 381-388, 2001.

Zhang, H., Ai, G., Yan, X., Li, W., and Liu, Y: Evolution of Vector Magnetic Field and White-Light Flares in a Solar Active Region (NOAA 6659) in 1991 June, Ap. J., 423, 828-846, 1994.

Zhang, J., Wang, J., Deng, Y., and Wu, D.: Magnetic Flux Cancellation Associated with the Major Solar Event on 2000 July 14, Ap. J., 548, L99-L102, 2001.

Zirin, H. and Marquette, W.: BEARALERTS - A successful flare prediction system, Sol. Phys., 131, 149-164, 1991.

Zirin, H. and Wang, H.: Narrow lanes of transverse magnetic field in sunspots, Nature, 363, 426-428, 1993. 